\title{
Reply to: "Include desensitization to radio contrast media in the diagnostic algorithm"
}

\author{
Knut Brockow ${ }^{1}$ \\ ${ }^{1}$ Technical University of Munich
}

August 28, 2020

I thank Dres. Alnaes and Helnes Bergen for their stimulating comment on my medical algorithm on the Diagnosis and Treatment of Radiocontrast Media Hypersensitivity. ${ }^{1}$ In their comment, they raised attention to the possible addition of desensitization to radiocontrast media ( $\mathrm{RCM}$ ) management, which was not depicted in the algorithm. ${ }^{2}$ I have been well aware of several reports on desensitization and have already discussed them in a previous paper, however commented there that "successful desensitization of RCM has been reported for immediate hypersensitivity reactions to RCM, but it is only used anectodically" and concluded not to include this procedure into the algorithm. ${ }^{3}$

In addition to the two papers on desensitization to RCM cited by Dr. Alaes, also a handful other cases have been published, some of them older. To my knowledge, at least as far as I can access these case reports, in none of these patients a proper allergy diagnosis and management has been performed and in most, if not all of these patients, desensitization probably was unnecessary. In the described cases, skin testing has not been performed or was even negative indicating a higher probability for a non-allergic immediate hypersensitivity reaction (IHR), in the history before desensitization was performed in several cases the RCM was not changed, but the same not tolerated RCM was given again and radiologists in vain relied on premedication to prevent recurrent attacks, and no skin test-negative RCM was identified and used. None of the cases published convinced me of the need for desensitization. Performing the examination with a skin test-negative RCM would with a high probability be successful. ${ }^{4}$ I would expect the success of desensitization was rather due to changing to a different isoosmolar RCM (and probably not to adding premedication) than the desensitization procedure itself, as alone changing the implicated RCM to another one in one study reduced the risk of recurrent IHR by $67.1 \%$ (odds ratio: $0.329 ; \mathrm{P}=0.001$ ), whereas steroid premedication did not show protective effects. ${ }^{5}$

Our group of European Network on Drug Allergy experts have highlighted that rapid desensitization is a procedure that can be used to provide a temporary tolerance to a first-line drug when no alternative is available. ${ }^{6}$ This implies for RCM hypersensitivity that using a skin-test-negative RCM for the next examination as an alternative drug is next step and not immediate desensitization. One problem with desensitization is that too many doctors employ it uncritically and without prior proper allergy workup, best with drug provocation test. The high rate of successful desensitizations without prior confirmation of drug hypersensitivity in the literature is in part explained by the fact that many of those patients would not have reacted anyway. I have yet to find convincing evidence to add desensitization as a standard therapeutic option to the RCM management algorithm.

Having said this, I am eagerly following up the literature on RCM desensitization with great interest to be prepared, should I encounter an own patient, who would react severely to an alternative skin test-negative RCM after following the algorithm. Until now, colleagues and I have not met such a patient, however, I would seriously consider desensitization as an option in such a situation. Thus, I thank Dres. Alnaes and Helsen Bergen for bringing up that interesting topic for discussion. 


\section{References}

1. Brockow K. Medical Algorithm: Diagnosis and Treatment of Radiocontrast Media Hypersensitivity. Allergy 2020; 75:1278-80.

2. Alnaes M, Helsen Bergen A. Include desenzitation to radiocontrast media in the diagnostic algorithm. Allergy 2020; To be determined!

3. Schonmann C, Brockow K. Adverse reactions during procedures: Hypersensitivity to contrast agents and dyes. Ann Allergy Asthma Immunol 2020; 124:156-64.

4. Trautmann A, Brockow K, Behle V, Stoevesandt J. Radiocontrast Media Hypersensitivity: Skin Testing Differentiates Allergy From Nonallergic Reactions and Identifies a Safe Alternative as Proven by Intravenous Provocation. J Allergy Clin Immunol Pract 2019; 7:2218-24.

5. Park HJ, Park JW, Yang MS, Kim MY, Kim SH, Jang GC, et al. Re-exposure to low osmolar iodinated contrast media in patients with prior moderate-to-severe hypersensitivity reactions: A multicentre retrospective cohort study. Eur Radiol 2017; 27:2886-93.

6. Cernadas JR, Brockow K, Romano A, Aberer W, Torres MJ, Bircher A, et al. General considerations on rapid desensitization for drug hypersensitivity - a consensus statement. Allergy 2010; 65:1357-66. 